\title{
Nonphotorealistic Virtual Environment Navigation from Images
}

\author{
Hyung W. Kang \\ Department of Mathematics and Computer Science \\ University of Missouri - St. Louis \\ One University Blvd. St. Louis, MO 63121, USA \\ Phone: 314-516-5841, Fax: 314-516-5400 \\ kang@cs.umsl.edu
}

\begin{abstract}
This paper presents a novel scheme based on a hybrid NPR/IBMR approach, for providing non-photorealistic virtual environment navigation from images. As a preprocess, we first divide the background and the foreground components in the image and then construct a simple $3 D$ scene model consisting of background model and foreground object models, together with the corresponding nonphotorealistic texture maps. Thus, through interactive navigation at runtime, non-photorealistic walk-through images for novel viewpoints can be generated in real time. To reduce visual artifacts in the resulting animation, we propose an effective method for generating multiresolution and view-dependent non-photorealistic texture maps that preserve the stroke size and coherence across different viewing resolutions and angles.
\end{abstract}

Keywords: Image-based modeling and rendering; Nonphotorealistic rendering; Virtual environment navigation

\section{Introduction}

\subsection{Motivation}

Providing interactive navigation in a virtual environment is one of the key issues in computer graphics. While most of the previous techniques have focused on generating photorealistic walk-through images, non-photorealistic rendering (NPR) has recently emerged as a meaningful alternative since it has clear advantages over photorealism. For example, a single scene can be expressed in various ways through artistic rendering, conveying the different mood or interpretation. Also, NPR can focus the viewer's attention on important features or semantics in the scene through abstraction or exaggeration.
However, conventional NPR techniques based on runtime placement of individual strokes are not suitable for interactive scene navigation since they require considerable time to create a single frame. One way to provide NPR in real time is to borrow the idea of image-based modeling and rendering (IBMR), that is, to create an image viewed from an arbitrary point by employing a set of reference photographs or pre-rendered images. This type of hybrid NPR/IBMR approach can provide artistic effects together with visual details of the scene in interactive rate. In this case, however, it is hard to preserve the stroke size and coherence across different resolutions and view angles, which is essential for producing convincing results.

Based on the hybrid NPR/IBMR approach, this paper presents a novel scheme for providing non-photorealistic virtual environment navigation from images. In the preprocessing stage, we extract the foreground objects from the background in the image and generate corresponding nonphotorealistic texture maps for them. Then we construct a simple 3D scene model which we use for generating nonphotorealistic walk-through images in runtime. Especially, our focus in this paper is how to generate non-photorealistic texture maps that have desirable properties. To minimize visual artifacts and to produce convincing effects in the resulting animation, we propose a novel idea for generating multiresolution and view-dependent non-photorealistic texture maps that preserve both the stroke size and coherence across different viewing resolutions and angles.

\subsection{Related work}

Various techniques have been developed for generating non-photorealistic or artistic effects such as painterly rendering $[5,12,6,20]$, pen and ink illustration [18, 19], watercolor painting [3], pencil drawing [21], ornamentation [22], engraving [16], and so on. Most of these techniques require 2D still images or patterns from which to obtain the spe- 
cific artistic style of interest. Thus, simply applying them to time-changing image sequences may create noisy, flickery results. While other approaches based on 3D models can solve this problem, they either lack visual details for exploiting only the information obtained from crude 3D models $[13,4,11]$, or take considerable time in creating a single frame due to the individual stroke maintenance process at run time [14].

Some researchers suggested a hybrid NPR/IBMR approach, by which we can obtain the temporal coherency, visual details, and interactive frame rate, simultaneously [7, 10, 2]. Especially, Klein et al. presented an idea for non-photorealistic virtual environment navigation based on conventional texture mapping [10]. They introduced nonphotorealistic version of mip-maps (called art-maps) to preserve the size of the strokes across different viewing resolutions and angles. However, their algorithm places strokes independently at each image level, and hence it does not provide any stroke coherence when the resolution of the texture map or the view angle is altered during navigation. The lack of stroke coherence can cause undesirable visual artifacts in the resulting walk-through image sequence.

\subsection{Overview}

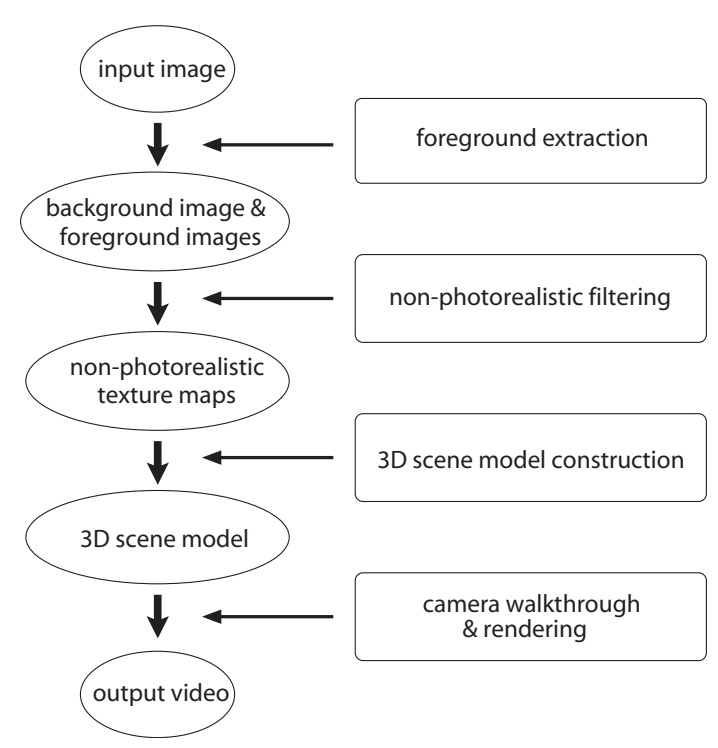

\section{Figure 1. Process flow diagram of non- photorealistic TIP}

Fig. 1 shows the process flow diagram of our entire scheme for generating non-photorealistic walk-through animation from an image, which is an extension of our previous work on TIP (Tour Into the Picture) [8]. TIP is a method for generating a sequence of walk-through images from a single reference image. Given an input image, we first extract the foreground objects from the background and set their corresponding bounding polygons. The resulting foreground texture images are alpha-masked on the exact portion of the objects using our highly interactive segmentation tool called Enhanced Lane [9], to produce convincing $3 \mathrm{D}$ effects during navigation (see Fig. 2b). The background texture image is then generated after filling in the holes created by the extracted foreground objects, by adopting inpainting techniques [1] (see Fig. 2c). Since our foreground extraction processes including object selection, boundary segmentation, hole filling, are all highly interactive, various different results can be obtained from the same input image, reflecting the animator's intentions.

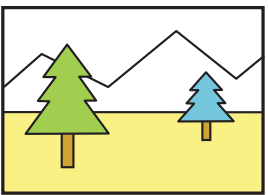

(a) input image (b) foreground images

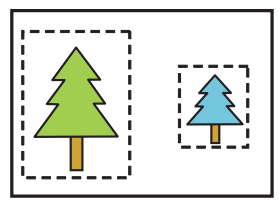

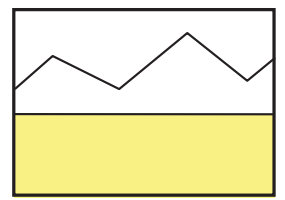

(c) background image

\section{Figure 2. Foreground extraction}

The background and the foreground images are then converted into non-photorealistic texture maps by applying various artistic filters. Basically, our scheme is independent of the filtering methods and does not stick to specific texture patterns or styles, and any filter can be used for producing appropriate non-photorealistic texture maps. However, not all filters ensure desirable properties such as stroke size or coherence preservation in the output images during navigation. Thus, we develop an algorithm to construct the nonphotorealistic version of mip-maps (called art-maps) which preserve the stroke size and the stroke coherence across different levels of texture resolution caused by camera movement. The key idea behind this is to form a nested structure of the strokes among different resolutions. The temporal coherence among strokes in a single level is automatically ensured since the novel view generation is based on conventional texture mapping. Also, we extend this idea to generate view-dependent texture maps so that these properties are preserved regardless of the camera view angles with respect to the object surface.

After texture image generation, we construct the $3 \mathrm{D}$ scene model which consists of a background model and foreground models. As shown in Fig. 3a and 3b, the background model is constructed based on the location of a vanishing line detected in the background image [8]. The foreground objects are then modeled as polygons and attached to the background model after computing their corresponding coordinates in 3D space (see Fig. 3c). With the constructed scene model and all the texture images prepared, we can navigate the virtual environment by interac- 
(a) $2 \mathrm{D}$ image

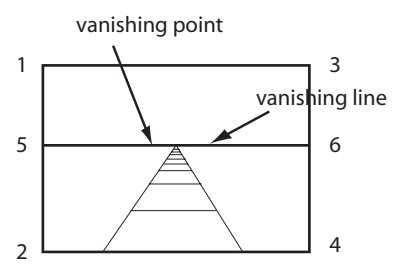

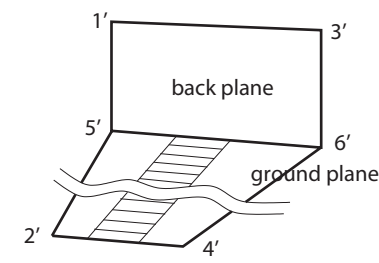

(b) 3D background model

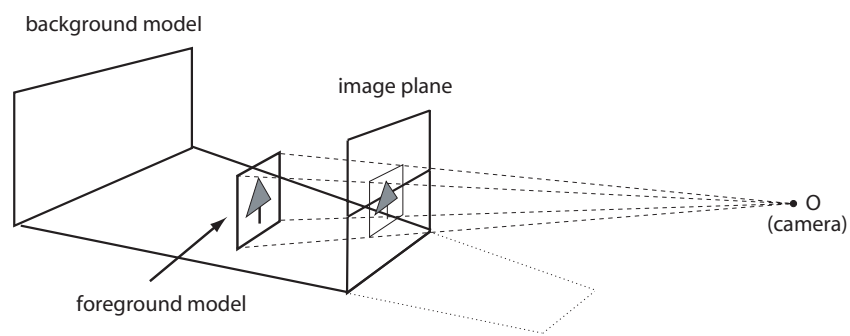

(c) 3D foreground model

Figure 3. Scene model construction

tively positioning the camera and successively creating nonphotorealistic images viewed from new viewpoints. Each frame in the output animation is generated by rendering the 3D scene model based on hardware texture mapping. The remainder of this paper is organized as follows. In Section 2, we explain the basics of our stroke placement technique. Then we discuss the method for generating nonphotorealistic mip-maps in Section 3, and show how to extend this idea to construct a view-dependent set of texture maps in Section 4. Section 5 provides some experimental results and Section 6 concludes this paper.

\section{Stroke placement strategy}

In this section, we explain our stroke placement algorithm. A stroke is defined as a rectangular area placed in an image, which has attributes such as color, orientation, length, width, etc. Various methods have been suggested for assigning the stroke attributes and each of them has its own style and advantages $[5,12,6,20]$. While any of these methods can be used for our application, we follow the convention of [12] which nicely produces the standard painterly effects. That is, given a pixel location $(x, y)$, the color is copied from the input image pixel at this location, the orientation follows the gradient direction (which is perpendicular to the gradient vector), the width is selected between the minimum and the maximum value, and the length is clipped by the edges with strong gradient magnitudes. Using all of these attributes, we draw a stroke centered at the given pixel location. These attributes can also be slightly perturbed in a predefined range to generate more hand-crafted look.
Another important issue is how to decide where and in which order we place the strokes. To construct multiple levels of texture resolutions as a nested structure, we use an optimization-style framework. Given an input image and a predefined range of stroke size, we want to generate a non-photorealistic output image which is as close to the input image as possible after placing a sufficient number of strokes. Thus, our goal is to minimize the following objective function:

$$
f\left(I^{\prime}\right)=\sum_{\mathbf{x}}\left\|I^{\prime}(\mathbf{x})-I(\mathbf{x})\right\|^{2}
$$

where $I$ and $I^{\prime}$ respectively denote input and output image, and $\mathbf{x}=(x, y)$ denotes each pixel coordinate.

Like an artist, we place individual strokes one by one on an output canvas (image) which is initialized as a white image. Before placing each stroke, we first test if it reduces the objective function in Equation 1, and place only the strokes that pass the test. The current stroke placing location is selected among the pixels with the biggest difference value $d(\mathbf{x})=\left\|I^{\prime}(\mathbf{x})-I(\mathbf{x})\right\|$ in the image. For efficient handling of the stroke placement order, we apply a bucket sort algorithm over the entire image pixels with respect to the difference value. Since the difference value can be discretized in a predefined integer range, we can maintain the active list in just $O(N)$ time where $N$ is the number of pixels [15]. Fig. 4 shows an example of our stroke placement process. The numbered pixels in Fig. 4a are the first three pixels with the highest priorities in the active list. As shown in Fig. 4d, a stroke can be partially (or completely) occluded by neighboring strokes, which is quite natural in painting.

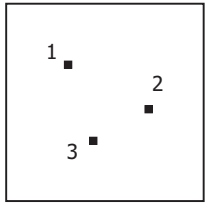

(a) Input image

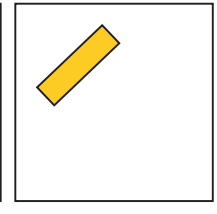

(b) First stroke

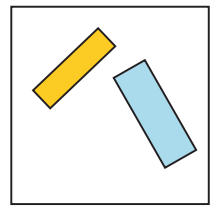

(c) Second stroke

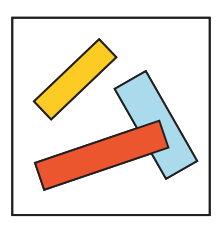

(d) Third stroke

Figure 4. Stroke placement

To accelerate the process and to produce more natural hand-crafted look, we first initialize the active list by randomly placing a sufficient number of strokes over the image, so that all the pixels can be covered by one or more stokes. Then the next pixel to place a stroke at is always picked among the candidates with the maximum difference value in the active list. If the pixel passes the validity test we place a stroke there and update the list, otherwise we simply discard the pixel from the list. While our stroke placement algorithm eventually terminates when there is no more pixel left in the active list, we rather set a threshold to control the termination point. That is, we stop the process if the maximum difference value of the current candidate pixel in the 
active list gets smaller than the specified threshold. Fig. 5 shows output images obtained by applying our stroke placing algorithm with different threshold values. ${ }^{1}$

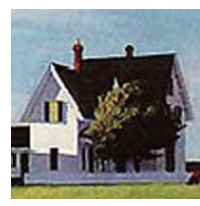

(a) input image

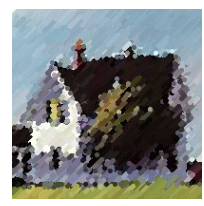

(b) threshold $=0.2$

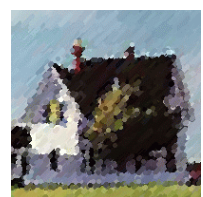

(b) threshold $=0.1$

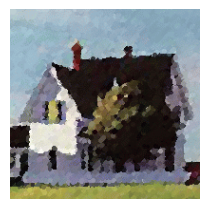

(b) threshold $=0.02$
Figure 5. Stroke optimization with different thresholds

\section{Constructing multiresolution maps}

If we use just a single level of texture maps, texture mapping hardware automatically resamples those maps at rendering so that the number of texture samples matches that of the current pixel resolution covered by the object of interest. This means that the closer the camera gets to the object, the bigger the size of the strokes becomes in the output image, which is undesirable in aesthetic point of view [14]. For this reason, we need to preserve the size of the stroke regardless of the camera position in that it can provide the consistent level of visual details during interactive walk-through. Klein et al. achieved this goal by introducing stroke-sizepreserving mip-maps, called art-maps [10]. Art-map is constructed by first lining up a set of multiresolution input images as in normal mip-mapping, then applying a nonphotorealistic filter to each level independently. Thus, at run time, the texture mapping hardware automatically selects the appropriate level in the mip-map hierarchy and blends the nearest two texture maps, so that the average stroke size remains almost the same wherever the camera is located.

However, since art-map is generated independently at each level, it lacks the stroke coherence across resolutions, which results in undesirable visual artifacts such as 'stroke swimming'. Inspired by [17], we design our nonphotorealistic mip-maps as a nested structure, such that all the strokes in a certain level are also contained in its higher resolution versions. Starting from the coarsest level, we apply our stroke placing algorithm to obtain corresponding non-photorealistic texture map at each level. We do not need to keep all the stroke attribute data to replicate them in the successive levels. Rather, at each stroke placement in a coarse level, we simultaneously place the samecolored stroke at the finer levels with successively doubling

\footnotetext{
${ }^{1}$ Note that even if we set the threshold value as 0 , the output image cannot be completely identical to the input image unless we predefine the stroke size to be as small as a single pixel.
}

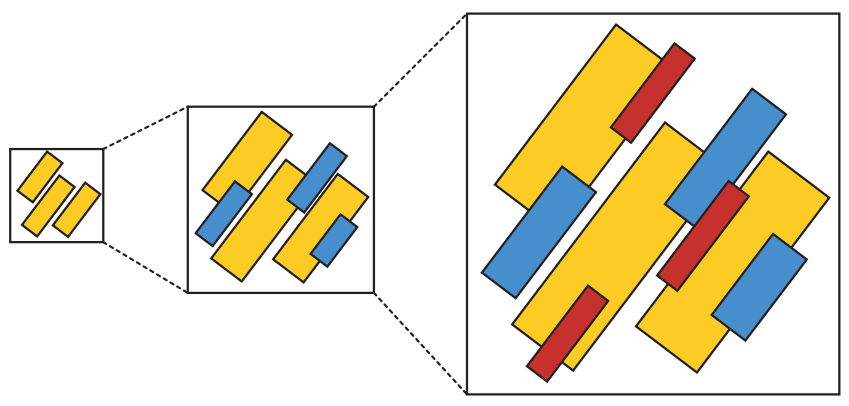

(a) Level 1

(b) Level 2

(c) Level 3
Figure 6. Nested structure of multiresolution strokes

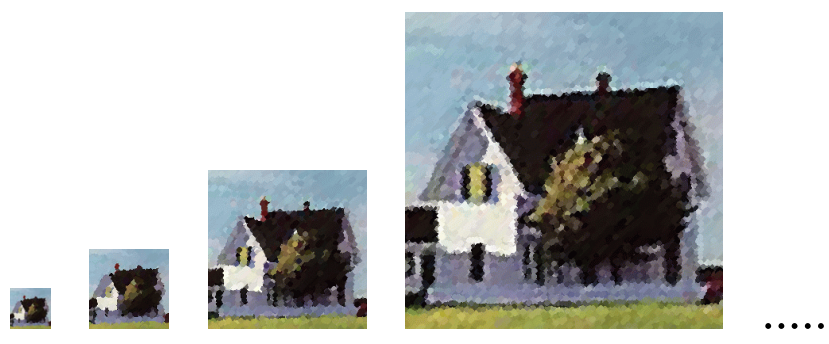

Figure 7. Non-photorealistic multiresolution texture maps

the width and length. After the optimization process is terminated for a current level, then we move on to the next finer level and start the process on the output image initialized by the previous level, using the main strokes with predefined size. Fig. 6 shows the nested structure of our multiresolution stroke placement method.

In this way, we can generate non-photorealistic mipmaps that keep the rules of resolution coherence and at the same time have been optimized at each level with the same size of strokes and threshold value. Since all the strokes in the previous level appear exactly at the same positions with doubled size in the current level, there can be no artifacts like swimming strokes. Also, since the main strokes of the predefined size are all placed in front of the big strokes inherited from the previous level, they dominate the overall impression of each level which is consistent in terms of stroke size across different resolutions. Fig. 7 shows some example non-photorealistic mip-maps generated by our technique.

\section{Constructing view-dependent maps}

The stroke size preservation problem occurs not only when the camera moves closer to an object, but also when 
the viewing direction forms an oblique angle with the object surface. As the angle gets more oblique, the size of the strokes gets smaller and their population becomes denser, which is visually undesirable. Also, the opposite case can happen due to the lack of sampled pixels in the input image, which is one of the typical problems of TIP (Tour Into the Picture) [7]. Since TIP obtains all the texture maps from small parts of the input image, each pixel (or stroke) should be unnaturally stretched out when the camera gets too close to an object or moves around the originally oblique surface, which degrades the realism in the output animation.
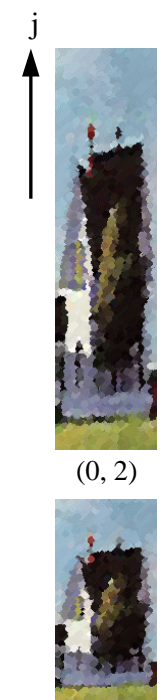

$(0,1)$

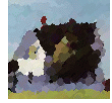

$(0,0)$

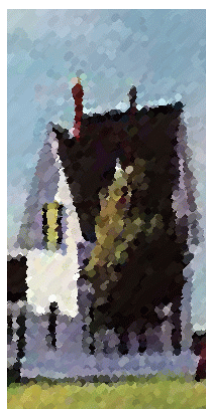

$(1,2)$

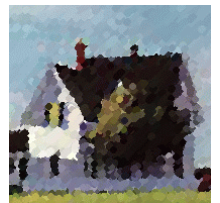

$(1,1)$

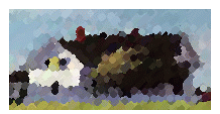

$(1,0)$

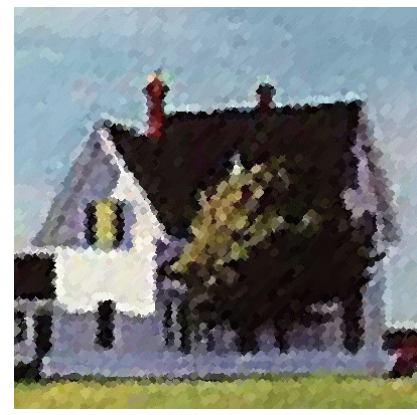

$(2,2)$

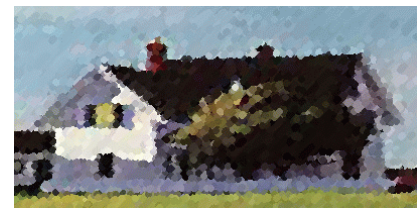

$(2,1)$

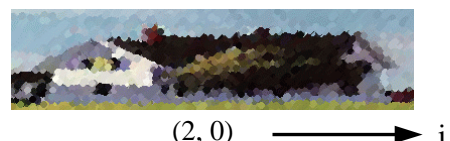

$(2,0)$

\section{Figure 8. View-dependent non-photorealistic texture maps}

To reduce this artifact, we generate view-dependent nonphotorealistic texture maps which provides an individual map for each viewing direction range (Fig. 8). To preserve the stroke size and coherence in this case also, we extend the idea used for generating non-photorealistic mip-maps. Starting from the coarsest image, we simultaneously place the strokes in the current image and the successive finer images, which are positioned along both the horizontal (i-axis) and the vertical (j-axis) directions. ${ }^{2}$ Once the stroke placing process is done for a current level, we move on and process the images in the next level, according to the following

\footnotetext{
${ }^{2}$ In case the original texture region does not contain a sufficient number of pixels, we can expand the texture map by bicubic interpolation and then apply our non-photorealistic filter. One of the merits of using nonphotorealistic texture maps is that we can avoid blurring artifacts even in an expanded image.
}

rules:

- Each texture map has its own $(i, j)$ index in the diagram (See Fig. 8).

- The level of each map is defined as $i+j$ where $(i, j)$ is the map index.

- The process starts from level 0 , that is, the coarsest map $(0,0)$.

- Every stroke placed in $(i, j)$ should also appear in all the finer maps $(m, n)$ where $m \geq i$ and $n \geq j$.

- If the process is done for all the maps in level $k$, then we move on to process the maps in the next level $k+1$.

- The whole process is terminated if all the maps have been optimized.

In this way, we can obtain a set of view-dependent nonphotorealistic texture maps which preserves both stroke size and coherence between them. At run time, we select an appropriate texture map from the set depending on the angle between the viewing direction and the object surface. ${ }^{3}$ Any in-between level of texture maps can be interpolated in real time by hardware multitexturing.

\section{Experimental results}

Fig. 9 shows the test result produced by our nonphotorealistic TIP scheme. The input image contains photorealistic scene with a lot of color variation and rich visual details. Fig. 9a is the input image and Fig. 9b is a stroke-optimized non-photorealistic output image. Fig. 9c and Fig. 9d show respectively the non-photorealistic versions of the interactive navigation shots taken from different camera locations. Note that even though different depth values are assigned for the background and the foreground objects, our non-photorealistic TIP scheme produces consistent level of stroke size and distribution over the output frames regardless of the camera position as shown in Fig. 9c and Fig. 9d. In Fig. 10, our scheme is applied to a nonphotorealistic input image. Using our stroke-based abstraction techniques, various new styles of walk-through images can be generated even from an already non-photorealistic image.

The rendering speed is dependent on the image size, the number of foreground objects, the number of resolution and view angle levels. In our current implementation, the entire scheme is implemented in $\mathrm{C}++$ with OpenGL library on Intel Pentium ${ }^{\mathrm{R}}$ PC (P4 2.20 GHz processor and $512 \mathrm{MB}$

\footnotetext{
${ }^{3}$ If the texture map was captured from an originally oblique surface in the input image, we should nullify the perspective correction in the rendering process to avoid double perspective effects [8].
} 


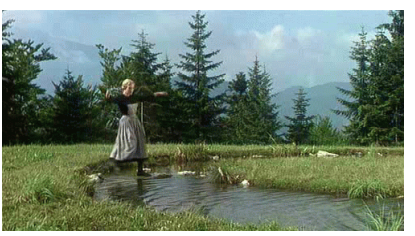

(a) Input image

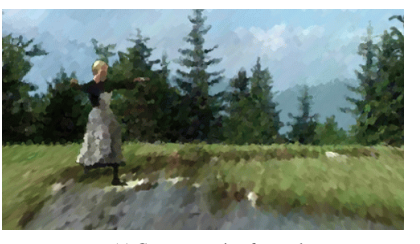

(c) Camera moving forward

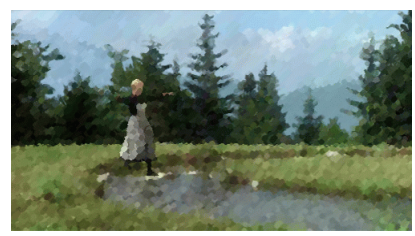

(b) Non-photorealistic view from initial camera

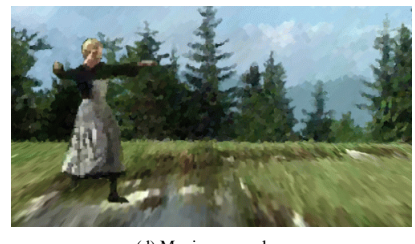

(d) Moving even close
Figure 9. Test on a photorealistic input image

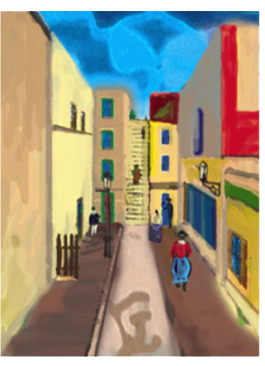

(a) Input image

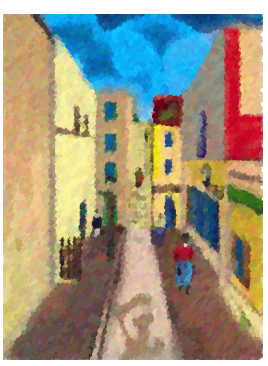

(b) From initial viewpoint

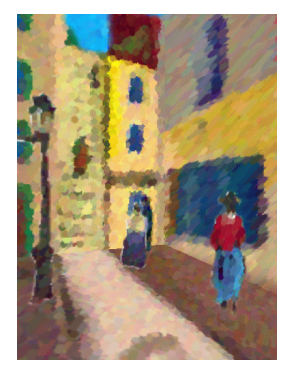

(c) From another viewpoint
Figure 10. Test on a non-photorealistic input image

memory) equipped with nVIDIA GeForce $3^{\mathrm{R}}$ graphics processor. On average, the output sequence of images with $640 \times 480$ pixels, less than 20 foreground objects, up to 10 levels of resolutions and view angles, is generated at an interactive rate (over 30 frames/sec).

\section{Conclusions and Future work}

In this paper, we have presented a novel scheme for generating non-photorealistic walk-through images from an input picture. Especially, our focus in this paper is to find a way to provide a consistent size and distribution of strokes throughout the output frames and maintain the coherence among them. For this purpose, we proposed a stroke placement algorithm based on optimization framework, together with a method for constructing multiresolution nonphotorealistic texture maps as a nested structure. We also extended this idea to generate a set of non-photorealistic view-dependent texture maps, so that the stroke size and coherence are preserved across different camera view directions. Given a single input image, the proposed scheme enables users to experience the non-photorealistic naviga- tion through artistically rendered scene in real time. Also, it can be used to solve some inherent problems in 3D virtual environment navigation based on conventional texture mapping, such as aliasing artifacts due to dense population of texture elements, or pixel stretching due to the insufficient number of sample texture pixels.

There are several aspects for further improvements. Although our scheme maintains the average stroke size and coherence in the output animation, the stroke size is not uniform on the object surface which is significantly warped in the output frame by perspective projection. Obviously, more texture maps (such as warped versions) should be added to the diagram in Section 4 to resolve this problem, in which case we would need a more efficient way to handle a huge set of texture maps. While we mostly used simple rectangular strokes in our current implementation, more sophisticated stroke types such as textured strokes [20] or curved strokes [6] would also work well in our scheme. Also, rather than oil painting or watercolor painting styles which can be covered by our algorithm, it would be challenging to provide different rendering styles such as penand-ink illustration $[18,19]$ or pencil drawing $[21]$ with a fully automated process. Finally, our scheme can be naturally extended to the panoramic images such as cylindrical or spherical images which may provide more immersive effects during camera navigation [8].

\section{Acknowledgment}

This research is supported by the MIC (Ministry of Information and Communication), Korea, under ITRC (Information Technology Research Center) support program supervised by IITA (Institute of Information Technology Assessment).

\section{References}

[1] M. Bertalmio, G. Sapiro, V. Caselles, and C. Ballester, "Image Inpainting", ACM SIGGRAPH 2000 Conference Proceedings, pp. 417-424, 2000.

[2] I. Buck, A. Finkelstein, C. Jacobs, A. Klein, D. Salesin, J. Seims, R. Szeliski, and K. Toyama, "Performance-driven hand-drawn animation", Proceedings of NPAR 2000, June, 2000.

[3] C. Curtis, S. Anderson, J. Seims, K. Fleischer, and D. Salesin, "Computer-generated watercolor", ACM SIGGRAPH 97 Conference Proceedings, pp. 421-430, 1997.

[4] A. Gooch, B. Gooch, P. Shirley, and E. Cohen, "A non-photorealistic lighting model for automatic tech- 
nical illustration”, ACM SIGGRAPH 98 Conference Proceedings, pp. 447-452, 1998.

[5] P. Haeberli, "Paint by numbers: Abstract image representations", ACM SIGGRAPH 90 Conference Proceedings, pp. 207-214, 1990.

[6] A. Hertzmann, "Painterly rendering with curved brush strokes of multiple sizes", ACM SIGGRAPH 98 Conference Proceedings, pp. 453-460, 1998.

[7] Y. Horry, K. Anjyo, and K. Arai, "Tour Into the Picture: Using a Spidery Mesh Interface to Make Animation from a Single Image", ACM SIGGRAPH 97 Conference Proceedings, pp. 225-232, 1997.

[8] H. Kang, S. Pyo, K. Anjyo, and S. Shin, "Tour Into the Picture using a Vanishing Line and its Extension to Panoramic Images", Computer Graphics Forum, 20(3), pp. 132-141, 2001.

[9] H. Kang and S. Shin, "Enhanced Lane: Interactive Image Segmentation by Incremental Path Map Construction”, Graphical Models, 64(5), pp. 282-303, 2002.

[10] A. Klein, W. Li, M. Kazhdan, W. Correa, A. Finkelstein, and T. Funkhouser, "Non-Photorealistic Virtual Environments", ACM SIGGRAPH 2000 Conference Proceedings, pp. 527-534, 2000.

[11] M. Kowalski, L. Markosian, J. Northrup, L. Bourdev, R. Barzel, L. Holden, and J. Hughes, "Art-based rendering of fur, grass, and trees", ACM SIGGRAPH 99 Conference Proceedings, pp. 433-438, 1999.

[12] P. Litwinowicz, "Processing images and video for an impressionist effect", ACM SIGGRAPH 97 Conference Proceedings, pp. 407-414, 1997.

[13] L. Markosian, M. Kowalski, S. Trychin, L. Bourdev, D. Goldstein, and J. Hughes, "Real-time nonphotorealtistic rendering", ACM SIGGRAPH 97 Conference Proceedings, pp. 415-420, 1997.

[14] B. Meier, "Painterly rendering for animation", $A C M$ SIGGRAPH 96 Conference Proceedings, pp. 477484, 1996.

[15] E. Mortensen and W. Barrett, "Intelligent Scissors for Image Composition", ACM SIGGRAPH 95 Conference Proceedings, pp. 191-198, 1995.

[16] V. Ostromoukhov, "Digital facial engraving", $A C M$ SIGGRAPH 99 Conference Proceedings, pp. 417424, 1999.

[17] E. Praun, H. Hoppe, M. Webb, and A. Finkelstein, "Real-Time Hatching", ACM SIGGRAPH 2001 Conference Proceedings, pp. 579-584, 2001.
[18] M. Salisbury, S. Anderson, R. Barzel, and D. Salesin, "Interactive pen-and-ink illustration", $A C M$ SIGGRAPH 94 Conference Proceedings, pp. 101108, 1994.

[19] M. Salisbury, M. Wong, J. Hughes, and D. Salesin, "Orientable textures for image-based pen-and-ink illustration”, ACM SIGGRAPH 97 Conference Proceedings, pp. 401-406, 1997.

[20] M. Shiraishi and Y. Yamaguchi, "An Algorithm for Automatic Painterly Rendering based on Local Source Image Approximation", Non-Photorealistic Animation and Rendering 2000 (NPAR 'O0), Annecy, 2000.

[21] Mario Costa Sousa and John W. Buchanan, "Computer-Generated Graphite Pencil Rendering of 3D Polygonal Models", Computer Graphics Forum, 18(3), 1999.

[22] M. Wong, D. Zongker, and D. Salesin, "Computergenerated floral ornament", ACM SIGGRAPH 98 Conference Proceedings, 19987. 